\title{
$O$ enriquecimento ambiental sobre o bem-estar de frangos de corte
}

\author{
Environmental enrichment on the welfare of industrial broiler chickens
}

\author{
Elaine Cristina de Oliveira Sans ${ }^{\mathrm{I}}$ Juliana Freitas Federici ${ }^{\mathrm{I}}$ Janaina Hammerschmidt $^{\mathrm{I}}$

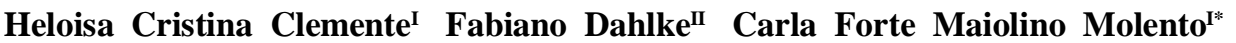

\section{RESUMO}

O objetivo deste trabalho foi avaliar os efeitos do enriquecimento ambiental sobre o bem-estar de frangos de corte em sistemas industriais. Um total de 400 frangos de corte machos, criados de um a 38 dias de idade, foram alojados em 20 boxes de $2 m^{2}$ com 20 aves unidade ${ }^{-1}$ experimental, sendo 10 boxes com ambiente controle (AC) e $10 \mathrm{com}$ ambiente enriquecido (AE) com poleiros, caixa de areia, couve, repolho e objetos suspensos. Aos sete, 21 e 35 dias foram avaliados claudicação, desvio valgo-varo e pododermatite (indicadores sanitários) e colhidas amostras sanguíneas. Na primeira, segunda e sexta semanas, o comportamento das aves foi registrado pelo método de varredura durante 13 horas/dia, a cada 20min. Semanalmente, foram avaliados consumo médio, ganho de peso médio e conversão alimentar (desempenho) das aves. Resultados hematológicos e de desempenho foram comparados pelo teste $F$, comportamentos $e$ indicadores sanitários pelo teste Chi quadrado e mortalidade pelo teste T. Não houve diferença entre tratamentos para indicadores sanitários. Não houve diferença para relação heterófilo:linfócito

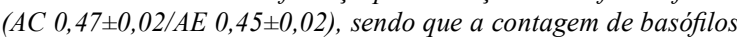
ficou acima da normalidade em ambos os tratamentos (1338 \pm 97 $\left.\mu L^{-1} ; 1212 \pm 106 \mu L^{-1}\right)$. Houve diminuição no comportamento de acocorar $(35,0 \% / 31,8 \%)$ e dormir $(14,6 \% / 13,1 \%)$ e aumento do banho de areia $(0,9 \% / 1,5 \%)$, bicar objetos $(0,2 \% / 0,6 \%)$, manutenção corporal $(12,7 \% / 14,2 \%)$ e forragear $(8,2 \% / 12,0 \%)$ em AE. Não houve diferença para mortalidade $(2,5 \% / 4,0 \%) e$ desempenho. Os itens de enriquecimento mostraram benefícios em relação ao comportamento das aves, mas problemas sanitários afetaram negativamente seu bem-estar em ambos os tratamentos.

Palavras-chave: comportamento, desempenho, dermatite de contato, heterófilo:linfócito.

\section{ABSTRACT}

The objective of this research was to evaluate the effects of environmental enrichment on the welfare of industrial broiler chickens. Four hundred male broilers, from one to 38 days, were equally distributed in 20 pens of $2 \mathrm{~m}^{2}$, being 10 pens controlled no-enriched boxes (CE) and 10 in environment enriched $(E E)$ with perches, sand tray, cabbage, kale and hanging objects. At 7, 21 and 35 days birds were individually scored for lameness, valgus-varus deviation and foot pad dermatitis (health indicators). Blood was collected form hematological analyses. At week one, two and six, in two boxes/treatment, behavior was assessed during 13 hours per day, using the scanning method, every 20min. Average consumption, weight gain and feed conversion (performance indicators) of broilers were weekly assessed. Hematologic and performance results were compared by $F$ test, behavior and health indicators by Chi square and mortality by $T$ test. There was no difference between enriched and no-enriched boxes for health indicators. There was no difference for the heterophil:lymphocyte ratio $(C E$ $0.47 \pm 0.02 / E E \quad 0.45 \pm 0.02$ ), but the basophil count was higher than normal values in both treatments $\left(1338 \pm 97 \mu L^{-1} ; 1212 \pm 106\right.$ $\left.\mu L^{-1}\right)$. There was a decrease in squatting (35.0\%/31.8\%) and sleeping $(14.6 \% / 13.1 \%)$ behaviors and increases in dust-bathing $(0.9 \% / 1.5 \%)$, pecking objects $(0.2 \% / 0.6 \%)$, body maintenance $(12.7 \% / 14.2 \%)$ and foraging $(8.2 \% / 12.0 \%)$ for enriched boxes. No significant difference in mortality $(2.5 \% / 4.0 \%)$ and performance were found. The enriched boxes showed benefits on chicken's behavior, however, health problems affected negatively the welfare of broiler chickens in enriched and no-enriched treatments.

Key words: behavior, contact dermatitis, heterophil:lymphocyte, performance.

\section{INTRODUÇÃO}

Frangos de corte são mantidos em sistemas com oportunidades restritas de escolhas ambientais. As opções de escolha dos animais podem ser incrementadas por meio do aumento da complexidade

\footnotetext{
'Laboratório de bem-estar animal (LABEA), Universidade Federal do Paraná (UFPR), 80035-050, Rua dos Funcionários, 1540, Juvevê, Curitiba, PR, Brasil. E-mail: carlamolento@ufpr.br.*Autor para correspondência.

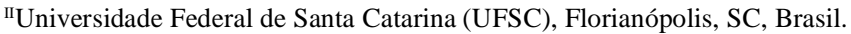


do ambiente, atingindo assim objetivos de bem-estar (BIZERAY et al., 2002a). Tornar o ambiente complexo e estimular a expressão de comportamentos naturais podem ser alcançados por meio da adoção de técnicas de enriquecimento ambiental (COSTA et al., 2012).

Diversos indicadores podem ser utilizados para avaliar a eficácia do enriquecimento. Alterações fisiológicas podem ser mensuradas por meio da contagem de células de defesa no sangue, sendo a relação heterófilo:linfócito $(\mathrm{H}: \mathrm{L})$ um indicador confiável de estresse crônico em frangos de corte (GROSS \& SIEGEL, 1983). O estado físico das aves pode ser comprometido pela presença de claudicação, desvios valgo-varo e dermatites de contato. A presença de um dos itens citados pode estar relacionada à dor no membro afetado, comprometendo a interação das aves com os recursos disponíveis no ambiente (SANOTRA et al., 2001; SHEPHERD \& FAIRCHILD, 2010).

A avaliação comportamental também faz parte do diagnóstico de bem-estar. Embora as aves tenham sido domesticadas e mantidas em ambientes confinados, raças modernas ainda carregam características comportamentais de seus ancestrais (DAWKINS, 1999). Considerando-se os potenciais benefícios do enriquecimento ambiental para a qualidade de vida dos animais, o objetivo deste trabalho foi avaliar os efeitos do enriquecimento sobre o bem-estar de frangos de corte em sistemas industriais.

\section{MATERIAL E MÉTODOS}

Foram utilizados 400 frangos de corte machos, linhagem Cobb $500^{\circledR}$, criados de um a 38 dias de idade, de novembro a dezembro de 2010 , na cidade de Videira/SC. As aves foram alojadas em boxes de $2,0 \mathrm{~m}^{2}$, com cama de maravalha nova. Os boxes foram equipados com comedouro tubular e bebedouro tipo nipple, em densidade de 10 aves $\mathrm{m}^{-2}$. Foi utilizado delineamento experimental inteiramente casualizado, com dois tratamentos e dez repetições com 20 aves por unidade experimental, sendo um tratamento em ambiente controle (AC) e outro em ambiente enriquecido (AE). Os itens de enriquecimento foram poleiros, caixa de areia, couve e repolho e itens suspensos como latas amassadas, tampas de garrafas e bolas coloridas. Os poleiros utilizados até os 21 dias apresentavam altura única de $10 \mathrm{~cm}$, comprimento de $45 \mathrm{~cm}$ e diâmetro de $1,5 \mathrm{~cm}$; no período de 21 a 38 dias, a altura variou de 10 a $40 \mathrm{~cm}$, comprimento de $60 \mathrm{~cm}$ e diâmetro de $3,5 \mathrm{~cm}$ (ESTEVEZ et al., 2002; STRUELENS et al., 2009). A caixa de areia era de alumínio, com diâmetro de
$40 \mathrm{~cm}$. O alimento verde e os itens suspensos foram alternados semanalmente. A temperatura do galpão foi aferida durante o período experimental por meio de termômetro de máxima e mínima e deste resultado foi gerada uma temperatura média para cada dia e semana. Foi fornecido aquecimento a gás para as aves até 14 dias de idade, a dieta foi fornecida ad libtum e a iluminação foi natural.

Aos sete, 21 e 35 dias de idade, em 40 aves tratamento ${ }^{-1}$, foram observados claudicação, desvio valgo-varo e pododermatite (JONES et al., 2005) (indicadores sanitários). Para claudicação, considerou-se ausência quando as aves caminharam normalmente, com equilíbrio e passos regulares e presença para qualquer alteração no padrão normal de andadura; para desvio valgo-varo, ausência para aves com ângulo de $180^{\circ}$ na articulação do tarso e presença para qualquer desvio valgo ou varo; para pododermatite, ausência para aves sem lesões e presença para qualquer diâmetro ou profundidade de lesão nas patas.

Foram coletadas amostras de sangue pela veia braquial de outras 40 aves tratamento ${ }^{-1}$. Aos sete dias, gotas de sangue foram coletadas para realização de esfregaço sanguíneo e determinação da relação H:L. Aos 21 e 35 dias, foi coletado $1 \mathrm{~mL}$ de sangue em tubos heparinizados, para hemograma. As contagens totais de eritrócitos e leucócitos foram realizadas em câmara de Neubauer com sangue diluído em Azul de Cresil brilhante a $0,05 \%$. Para a diferenciação dos leucócitos, foram realizados esfregaços sanguíneos. As lâminas foram coradas com corante de Wright e 100 células foram analisadas em microscópio óptico com aumento de 100 vezes. O hematócrito foi determinado em amostra centrifugada a 12000rpm por 5min. A concentração de hemoglobina foi avaliada pelo método colorimétrico por cianeto de hemoglobina na proporção de $2 \mathrm{~mL}$ de reagente para $20 \mu \mathrm{L}^{-1}$ de amostra de sangue.

O etograma baseou-se em dois dias semanais de observação comportamental. Durante a primeira semana, foram observados dois boxes por tratamento e, da segunda a sexta semana, quatro boxes por tratamento. As observações foram efetuadas por um período total de $13 \mathrm{~h} \mathrm{dia}{ }^{-1}$, das 07 às $20 \mathrm{~h}$, pelo método de varredura, com registro instantâneo a cada 20min, em um total de 12 dias de observação. Os comportamentos foram: a) acocorar: corpo da ave em contato com a cama; b) alerta: ave parada, em pé; c) beber água: consumir água do bebedouro; d) bicar não agressivo: bicar levemente outra ave; e) bicar agressivo: bicar outra ave, provocando reação agressiva ou defensiva; f) bicar objetos: bicada 
direcionada para objetos ou parte do box, com exceção do comedouro e bebedouro; g) comer alimento verde: bicar folhas de couve ou repolho; h) comer ração: bicar a ração do comedouro; i) dormir: ave com os olhos fechados, acocorada ou empoleirada; j) empoleirar: subir ou estar no poleiro; k) esticar as pernas: esticar uma das pernas, num ângulo de $90^{\circ}$ com relação ao corpo; 1) forragear: procurar alimento utilizando as patas e/ou bico; m) locomover-se: ave andando ou correndo; m) manutenção corporal: esticar as asas e/ ou pernas, sacudir as penas, bater as asas, coçar-se ou examinar as penas; o) banho de areia: revolver-se no substrato da cama ou da caixa de areia.

As aves e a ração foram pesadas semanalmente para avaliação do consumo médio, ganho de peso médio e conversão alimentar (desempenho). A mortalidade foi observada diariamente. Resultados hematológicos e de desempenho foram comparados pelo teste $\mathrm{F}$ da Análise de Variância a 5,0\% de probabilidade, com utilização do teste de normalidade Shapiro Wilk a $5,0 \%$ de probabilidade, indicadores comportamentais e sanitários pelo teste Chi quadrado a 5,0\% de probabilidade e mortalidade pelo teste $\mathrm{T}$ a $5,0 \%$ de probabilidade.

\section{RESULTADOS E DISCUSSÃO}

A temperatura média observada encontrou-se fora do padrão recomendado em $71,0 \%$ do tempo. Fatores térmicos inadequados podem comprometer o desenvolvimento e bemestar das aves (DAMASCENO et al., 2010). Não foi observada diferença $(\mathrm{P}>0,05)$ entre os tratamentos para claudicação, desvio valgo-varo e pododermatite (Tabela 1). O desvio varo foi observado somente aos 35 dias em AC. SU et al. (2000) e BIZERAY et al. (2002b) também observaram que um ambiente enriquecido não foi suficiente para alterar a prevalência de claudicação em frangos de corte. GRANDIN (2009) recomenda que um lote deve manter $95,0 \%$ das aves capazes de caminhar 10 passos sem demostrar qualquer grau de claudicação. Para o desvio valgo-varo, BONAMIGO (2010) observou $48,0 \%$ de frangos com valgo, resultado similar ao encontrado neste trabalho para aves aos 35 dias de idade no tratamento $\mathrm{AC}(50,0 \%)$. Com relação à pododermatite, GRANDIN (2009) encontrou uma média de $18,8 \%$ das aves com algum tipo de lesão, resultado inferior ao observado neste trabalho em frangos com 35 dias de idade $(32,0 \%$ AC; $30,0 \%$ $\mathrm{AE})$. A inclusão de enriquecimento não diminuiu a prevalência dos indicadores sanitários.

A média e erro padrão para $\mathrm{H}: \mathrm{L}$ foram $0,47 \pm 0,02$ para $\mathrm{AC}$ e $0,45 \pm 0,02$ para $\mathrm{AE}(\mathrm{P}>0,05)$, dentro dos valores de referência (Tabela 2). Foi observado um aumento para eritrócitos, leucócitos, heterófilos, linfócitos, monócitos e eosinófilos $(\mathrm{P}<0,05)$, com o decorrer da idade das aves, coerente com a literatura (CLARK et al., 2009). Os números de basófilos foram $1338 \pm 97 \mu \mathrm{L}^{-1}$ para AC e $1212 \pm 106 \mu \mathrm{L}^{-1}$ para $\mathrm{AE}$, fora dos valores de referência, sugerindo que as aves foram submetidas a algum desafio. Altas temperaturas têm potencial de estressar os animais e alterar o número de basófilos (ALTAN et al., 2000). Ainda, GREENE et al. (1985) observaram a presença de basófilos na camada de queratina, durante a fase inicial de uma inflamação.

Tabela 1 - Número de frangos de corte e respectiva porcentagem de prevalência de claudicação, desvio valgo-varo e pododermatite, por tratamento, sendo AC ambiente controle e AE ambiente enriquecido.

\begin{tabular}{|c|c|c|c|c|}
\hline \multirow{2}{*}{ Indicadores } & \multirow{2}{*}{ Tratamento } & \multicolumn{3}{|c|}{-Idade das aves (dias) -- } \\
\hline & & 7 & 21 & 35 \\
\hline \multirow{2}{*}{ Com claudicação } & $\mathrm{AC}$ & $2(5,0 \%) \mathrm{Ab}$ & $3(7,5 \%) \mathrm{Ab}$ & $13(32,5 \%) \mathrm{Aa}$ \\
\hline & $\mathrm{AE}$ & $2(5,0 \%) \mathrm{Aa}$ & $4(10,0 \%) \mathrm{Aa}$ & $7(17,5 \%) \mathrm{Aa}$ \\
\hline \multirow{2}{*}{ Com desvio valgo-varo } & $\mathrm{AC}$ & $2(5,0 \%) \mathrm{Ac}$ & $11(27,5 \%) \mathrm{Ab}$ & $20^{*}(50,0 \%) \mathrm{Aa}$ \\
\hline & $\mathrm{AE}$ & $2(5,0 \%) \mathrm{Ab}$ & $8(20,0 \%) \mathrm{Ab}$ & $12(30,0 \%) \mathrm{Aa}$ \\
\hline \multirow{2}{*}{ Com pododermatite } & $\mathrm{AC}$ & $4(10,0 \%) \mathrm{Ab}$ & $3(7,5 \%) \mathrm{Ab}$ & $13(32,0 \%) \mathrm{Aa}$ \\
\hline & $\mathrm{AE}$ & $1(2,5 \%) \mathrm{Ab}$ & $1(2,5 \%) \mathrm{Ab}$ & $12(30,0 \%) \mathrm{Aa}$ \\
\hline
\end{tabular}

*observação do único frango de corte com desvio varo.

Em coluna, número de ocorrências seguidas da mesma letra maiúscula dentro do parâmetro avaliado não difere pelo teste Chi quadrado $(\mathrm{P}>0,05)$.

Em linha, número de ocorrências seguidas de mesma letra minúscula não difere pelo teste Chi quadrado $(\mathrm{P}>0,05)$. 
Tabela 2 - Média \pm erro padrão para eritrócitos, hematócrito, hemoglobina, leucócitos totais, heterofilos, linfócitos, monócitos, eosinófilos, basófilos e heterófilo:linfócito (H:L) de frangos de corte mantidos em dois tratamentos (Trat), sendo AC ambiente controle e AE ambiente enriquecido.

\begin{tabular}{|c|c|c|c|c|c|}
\hline \multirow{2}{*}{ Indicadores } & \multirow{2}{*}{ Trat } & \multicolumn{3}{|c|}{ 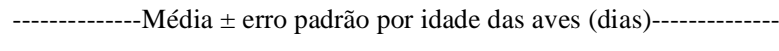 } & \multirow{2}{*}{--Referencia*-- } \\
\hline & & 7 & 21 & 35 & \\
\hline \multirow{2}{*}{ Eritrócitos (milhões $\mu \mathrm{L}^{-1}$ ) } & $\mathrm{AC}$ & - & $2,60 \pm 0,05 \mathrm{Aa}$ & $2,90 \pm 0,04 \mathrm{Ab}$ & \multirow{2}{*}{$2,50-3,50$} \\
\hline & $\mathrm{AE}$ & - & $2,60 \pm 0,06 \mathrm{Aa}$ & $2,81 \pm 0,03 \mathrm{Ab}$ & \\
\hline \multirow{2}{*}{$\begin{array}{l}\text { Hematócrito } \\
(\%)\end{array}$} & $\mathrm{AC}$ & - & $31,3 \pm 0,9 \mathrm{Aa}$ & $32,5 \pm 0,4 \mathrm{Aa}$ & \multirow{2}{*}{$22-35$} \\
\hline & $\mathrm{AE}$ & - & $30,9 \pm 0,6 \mathrm{Aa}$ & $32,0 \pm 0,3 \mathrm{Aa}$ & \\
\hline \multirow{2}{*}{ Hemoglobina $\left(g \mu^{-1}\right)$} & $\mathrm{AC}$ & - & $10,4 \pm 0,2 \mathrm{Aa}$ & $10,8 \pm 0,1 \mathrm{Aa}$ & \multirow{2}{*}{$7-13$} \\
\hline & $\mathrm{AE}$ & - & $10,3 \pm 0,2 \mathrm{Aa}$ & $10,6 \pm 0,1 \mathrm{Aa}$ & \\
\hline \multirow{2}{*}{ Leucócitos $\left(\mu \mathrm{L}^{-1}\right)$} & $\mathrm{AC}$ & - & $15400 \pm 906 \mathrm{Aa}$ & $21525 \pm 897 \mathrm{Ab}$ & \multirow{2}{*}{$12000-30000$} \\
\hline & $\mathrm{AE}$ & - & $14500 \pm 871 \mathrm{Aa}$ & $22300 \pm 1162 \mathrm{Ab}$ & \\
\hline \multirow{2}{*}{ Heterófilos $\left(\mu \mathrm{L}^{-1}\right)$} & $\mathrm{AC}$ & - & $4021 \pm 407 \mathrm{Aa}$ & $6107 \pm 516 \mathrm{Ab}$ & \multirow{2}{*}{$3000-6000$} \\
\hline & $\mathrm{AE}$ & - & $3719 \pm 412 \mathrm{Aa}$ & $5723 \pm 462 \mathrm{Ab}$ & \\
\hline \multirow{2}{*}{ Linfócitos $\left(\mu \mathrm{L}^{-1}\right)$} & $\mathrm{AC}$ & - & $9281 \pm 623$ Aa & $12986 \pm 690 \mathrm{Ab}$ & \multirow{2}{*}{$7000-17500$} \\
\hline & $\mathrm{AE}$ & - & $9010 \pm 531 \mathrm{Aa}$ & $14309 \pm 942 \mathrm{Ab}$ & \\
\hline \multirow{2}{*}{ Monócitos $\left(\mu \mathrm{L}^{-1}\right)$} & $\mathrm{AC}$ & - & $136 \pm 21 \mathrm{Aa}$ & $397 \pm 47 \mathrm{Ab}$ & \multirow{2}{*}{$150-2000$} \\
\hline & $\mathrm{AE}$ & - & $166 \pm 26 \mathrm{Aa}$ & $345 \pm 41 \mathrm{Ab}$ & \\
\hline \multirow{2}{*}{ Eosinófilos $\left(\mu \mathrm{L}^{-1}\right)$} & $\mathrm{AC}$ & - & $484 \pm 59 \mathrm{Aa}$ & $830 \pm 92 \mathrm{Ab}$ & \multirow{2}{*}{$0-1000$} \\
\hline & $\mathrm{AE}$ & - & $387 \pm 51 \mathrm{Aa}$ & $707 \pm 105 \mathrm{Ab}$ & \\
\hline \multirow{2}{*}{ Basófilos $\left(\mu \mathrm{L}^{-1}\right)$} & $\mathrm{AC}$ & - & $1473 \pm 146 \mathrm{Aa}$ & $1202 \pm 125 \mathrm{Aa}$ & \multirow{2}{*}{ raros } \\
\hline & $\mathrm{AE}$ & - & $1213 \pm 163 \mathrm{Aa}$ & $1211 \pm 138 \mathrm{Aa}$ & \\
\hline \multirow{2}{*}{$\mathrm{H}: \mathrm{L}$} & $\mathrm{AC}$ & $0,40 \pm 0,02 \mathrm{Aa}$ & $0,49 \pm 0,05 \mathrm{Aa}$ & $0,53 \pm 0,05 \mathrm{Aa}$ & \multirow{2}{*}{$0,30-1,70$} \\
\hline & $\mathrm{AE}$ & $0,44 \pm 0,03 \mathrm{Aa}$ & $0,46 \pm 0,05 \mathrm{Aa}$ & $0,46 \pm 0,04 \mathrm{Aa}$ & \\
\hline
\end{tabular}

"Valores de referência: BOUNOUS e STEDMAN, 2000

Em coluna, médias seguidas da mesma letra maiúscula não diferem pelo teste $\mathrm{F}(\mathrm{P}>0,05)$.

Em linha, médias seguidas da mesma letra minúscula não diferem pelo teste $F(P>0,05)$.

Sendo assim, uma infecção bacteriana pode alterar o número de basófilos (SHEPHERD \& FAIRCHILD, 2010), o que pode ter colaborado para os altos valores observados. Os valores hematológicos são sensíveis a diversos fatores, sendo que a variação da temperatura ambiente e a pododermatite aparentam ter relação com o aumento de basófilos.

Foi encontrada diferença $(\mathrm{P}<0,05)$ entre os tratamentos para os comportamentos de acocorar, banho de areia, beber água, bicar objetos, comer ração, dormir, manutenção corporal e forragear (Tabela 3). BIZERAY et al. (2002a) verificaram que aves mantidas em um ambiente com barreiras de madeira diminuíram a frequência do acocorar, pois as aves andavam mais até as fontes de alimento. $\mathrm{O}$ acocorar é utilizado para o descanso, sendo uma necessidade etológica importante para as aves (PICKEL et al., 2010). O aumento de banho de areia foi observado com maior frequência $(\mathrm{P}<0,05)$ em AE. Este comportamento é considerado positivo para as aves, pois é de alta motivação e auxilia na manutenção de uma boa condição de plumagem (EUROPEAN COMISSION, 2000). O bicar objetos foi observado com maior frequência $(\mathrm{P}<0,05)$ em $\mathrm{AE}$. HEIKKILÄ et al. (2006) penduraram CDs e garrafas de plástico para incentivar o empoleirar de frangos de corte, mas não encontraram resultados significativos. A manutenção corporal foi observada com maior frequência $(\mathrm{P}<0,05)$ em $\mathrm{AE}$, sendo considerado essencial para a integridade física das aves. $O$ forragear foi observado com maior frequência $(\mathrm{P}<0,05)$ em $\mathrm{AE}$, sendo um comportamento que compõe o repertório 
Tabela 3 - Número de observações comportamentais e respectiva porcentagem para frangos de corte criados de um a 38 dias de idade, em função do tratamento e efeito do período experimental (semanas), sendo AC ambiente controle e AE ambiente enriquecido.

\begin{tabular}{lll}
\hline & & \\
Comportamento & AC & AE \\
\hline Acocorar & $6113(35,0 \%) \mathrm{a}$ & $5306(31,8 \%) \mathrm{b}$ \\
Alerta & $240(1,4 \%)$ & $199(1,2 \%)$ \\
Banho de areia & $152(0,9 \%) \mathrm{b}$ & $255(1,5 \%) \mathrm{a}$ \\
Beber água & $1246(7,1 \%) \mathrm{a}$ & $1108(6,6 \%) \mathrm{b}$ \\
Bicagem não agressiva & $152(0,9 \%)$ & $162(1,0 \%)$ \\
Bicagem agressiva & $68(0,4 \%)$ & $78(0,5 \%)$ \\
Bicar objetos & $36(0,2 \%) \mathrm{b}$ & $104(0,6 \%) \mathrm{a}$ \\
Comer ração & $2379(13,6 \%) \mathrm{a}$ & $2119(12,7 \%) \mathrm{b}$ \\
Dormir & $2551(14,6 \%) \mathrm{a}$ & $2189(13,1 \%) \mathrm{b}$ \\
Esticar pernas & $76(0,4 \%)$ & $85(0,5 \%)$ \\
Forragear & $1438(8,2 \%) \mathrm{b}$ & $1996(12,0 \%) \mathrm{a}$ \\
Locomoção & $804(4,6 \%)$ & $728(4,4 \%)$ \\
Manutenção corporal & $2225(12,7 \%) \mathrm{b}$ & $2363(14,2 \%) \mathrm{a}$ \\
\hline
\end{tabular}

Médias seguidas de letras diferentes na mesma linha diferem pelo teste Chi quadrado $(\mathrm{P}>0,05)$.

alimentar das aves. CORNETTO \& ESTEVEZ (2001) observaram um aumento significativo de forragear na inclusão de painéis verticais, cujo objetivo foi gerar áreas adicionais no ambiente de criação de frangos, resultado similar ao encontrado neste estudo. Em linhas gerais, comportamentos importantes para a espécie foram mais prevalentes no tratamento AE. Entretanto, empoleirar foi observado somente $0,12 \%$ do tempo e comer alimento verde em $0,96 \%$. A alta incidência de claudicação, desvio valgo-varo e pododermatite pode ter influenciado no ato de empoleirar, pois dificulta a locomoção das aves (MACGEOWN et al., 1999; VESTERGAARD \& SANOTRA, 1999; SANOTRA, et al., 2001). As aves não expressaram interesse por alimento verde.
A observação de comportamentos positivos para a espécie está relacionada à melhoria no grau de bemestar (DUNCAN, 1998). Portanto, os resultados deste trabalho foram favoráveis para a expressão do repertório comportamental das aves.

Para os índices de consumo médio de ração, ganho de peso médio e conversão alimentar, não houve diferença $(\mathrm{P}>0,05)$, com exceção entre os dias 8-14 para o consumo médio de ração (Tabela 4). BIZERAY et al. (2002b) não observaram diferenças na conversão alimentar de frangos de corte entre ambiente controle e enriquecido. SIMSEK et al. (2009) não observaram significância no ganho de peso diário, conversão alimentar e consumo de ração de frangos em comparação entre ambientes estéreis e enriquecidos. A não interferência

Tabela 4 - Consumo médio de ração (CMR), ganho de peso médio (GPM) e conversão alimentar (CA) de frangos de corte em função da idade e tratamento (Trat), sendo AC ambiente controle e AE ambiente enriquecido.

\begin{tabular}{|c|c|c|c|c|c|c|c|}
\hline \multirow{2}{*}{ Indicadores } & \multirow{2}{*}{ Trat } & \multicolumn{6}{|c|}{--Idade das aves (dias) -- } \\
\hline & & $1-7$ & 8-14 & $15-21$ & $22-28$ & $29-35$ & $1-35$ \\
\hline \multirow{2}{*}{ CMR/ave (kg) } & $\mathrm{AC}$ & $0,156 \mathrm{f}$ & $0,391 \mathrm{Ae}$ & $0,698 \mathrm{~d}$ & $1,001 \mathrm{c}$ & $1,311 \mathrm{~b}$ & $3,559 \mathrm{a}$ \\
\hline & $\mathrm{AE}$ & $0,152 \mathrm{f}$ & $0,386 \mathrm{Be}$ & $0,711 \mathrm{~d}$ & $0,984 \mathrm{c}$ & $1,306 \mathrm{~b}$ & $3,542 \mathrm{a}$ \\
\hline \multirow{2}{*}{ GPM/ave (kg) } & $\mathrm{AC}$ & $0,160 \mathrm{f}$ & $0,311 \mathrm{e}$ & $0,512 \mathrm{~d}$ & $0,675 \mathrm{c}$ & $0,797 \mathrm{~b}$ & $2,454 \mathrm{a}$ \\
\hline & $\mathrm{AE}$ & $0,158 \mathrm{f}$ & $0,304 \mathrm{e}$ & $0,508 \mathrm{~d}$ & $0,674 \mathrm{c}$ & $0,806 \mathrm{~b}$ & $2,450 \mathrm{a}$ \\
\hline \multirow{2}{*}{ CA } & $\mathrm{AC}$ & $0,977 \mathrm{f}$ & $1,262 \mathrm{de}$ & $1,368 \mathrm{~d}$ & $1,485 \mathrm{c}$ & $1,656 \mathrm{~b}$ & $1,451 \mathrm{a}$ \\
\hline & $\mathrm{AE}$ & $0,963 \mathrm{f}$ & $1,269 \mathrm{e}$ & $1,405 \mathrm{~d}$ & $1,461 \mathrm{c}$ & $1,632 \mathrm{~b}$ & $1,447 \mathrm{a}$ \\
\hline
\end{tabular}

Em coluna, médias seguidas de letra maiúscula diferente, dentro do mesmo parâmetro avaliado, diferem pelo teste $\mathrm{F}(\mathrm{P}>0,05)$.

Em linha, médias seguidas de mesma letra minúscula não diferem pelo teste $\mathrm{F}(\mathrm{P}>0,05)$.

Ciência Rural, v.44, n.10, out, 2014. 
dos itens de enriquecimento sobre os índices zootécnicos não aparenta gerar prejuízos aos produtores, sendo ainda positivo ao bem-estar das aves. Não foram encontradas diferenças $(\mathrm{P}>0,05)$ para mortalidade no período de 1 a 35 dias de idade, sendo 2,5\% para $\mathrm{AC}$ e $4,0 \%$ para AE. Os resultados deste trabalho estão de acordo com a literatura, sendo possível afirmar que o enriquecimento não influenciou no desempenho das aves.

\section{CONCLUSÃO}

As técnicas de enriquecimento ambiental proporcionaram aumento da expressão do repertório comportamental dos frangos de corte, não influenciando os indicadores sanitários, hematológicos e de desempenho. Dessa forma, houve ganho de bem-estar animal pelo aumento da liberdade comportamental.

\section{AGRADECIMENTO}

E.C.O. SANS foi bolsista do Conselho Nacional de Desenvolvimento Científico e Tecnológico (CNPq).

\section{COMITÊ DE ÉTICA E BIOSSEGURANÇA}

Aprovado pelo Comitê de Ética no Uso de Animais da Universidade Federal do Paraná sob protocolo n.018/2010.

\section{REFERÊNCIAS}

ALTAN, O. et al. Effects of heat stress on some blood parameters in broilers. Turk Journal Veterinary Animal Science, v.24, p.145-148, 2000. Disponível em: <http://journals.tubitak.gov.tr/ veterinary/issues/vet-00-24-2/vet-24-2-8-9904-4.pdf>. Acesso em: 20 nov. 2011

BIZERAY, D. et al. Effects of increasing environmental complexity on the physical activity of broiler chickens. Applied Animal Behaviour Science, v.79, p.27-41, 2002a. Disponível em: <http://www.sciencedirect.com/science/article/pii/S01681 59102000837>. Acesso em: 01 nov. 2011. doi: 10.1016/S01681591(02)00083-7.

BIZERAY, D. et al. Influence of increased environmental complexity on leg condition, performance and level of fearfulness in broilers. Poultry Science, v.81, p.767-773, 2002b. Disponível em: <http://www.ncbi.nlm.nih.gov/pubmed/12079041>. Acesso em: 03 dez. 2011

BONAMIGO, A. Pontos selecionados de bem-estar para frangos de corte. 2010. 96f. Dissertação (Mestrado em Ciências Veterinárias) - Programa de Pós-graduação em Ciências Veterinárias. Universidade Federal do Paraná, Curitiba, PR.

BOUNOUS, D.I.; STEDMAN, N.L. Normal Avian Heamtology: Chicken and Turkey. In: FELDMAN, B.F.; ZINKL, J.G.; JAIN, N.C. Schalm's veterinary hematology. 5.ed. Philadelphia, Lippincott Williams \& Wilkins, 2000, p. 417-432.
CLARK, P. et al. Atlas of clinical avian hematology. Local: United Kingdom. Wiley-Blackwell, 2009. 184 p.

CORNETTO, T.; ESTEVEZ, I. Behavior of the domestic fowl in the presence of vertical panels. Poultry Science, v.80, p.14551462, 2001. Disponível em: <http://www.ncbi.nlm.nih.gov/ pubmed/11599705>. Acesso em: 12 set. 2011. doi: 10.1093/ ps/80.10.1455.

COSTA, L.S. et al. Some aspects of chicken behavior and welfare. Brazilian Journal of Poultry Science, v.14, n.3, p.159-164, 2012. Disponível em: <http://www.scielo.br/scielo. php?script $=$ sci_arttext $\&$ pid $=S 1516-635 X 2012000300001>$. Acesso em: 18 dez. 2012. doi: http://dx.doi.org/10.1590/S1516$635 \times 2012000300001$

DAMASCENO, F.A. et al. Avaliação do bem-estar de frangos de corte em dois galpões comerciais climatizados. Ciência Agrotécnica, v.34, n.4, p.1031-1038, 2010. Disponível em: <http://www.scielo.br/scielo.php?pid=S1413$70542010000400033 \&$ script=sci_arttext $>$. Acesso em: $15 \mathrm{dez}$. 2012. doi: http://dx.doi.org/10.1590/S1413-70542010000400033.

DAWKINS, M.S. The role of behaviour in the assessment of poultry welfare. World's Poultry Science Journal, v.55, p.295-303, 1999. Disponível em: <http://journals.cambridge. org/action/displayAbstract?fromPage $=$ online $\&$ aid $=621920>$. Acesso em: 20 dez. 2012. doi: http:/dx.doi.org/10.1079/ WPS 19990022.

DUNCAN, I.J.H. Behavior and behavioral needs. First North American Symposium on Poultry Welfare. Poultry Science, v.77, p.1766-1772, 1998. Disponível em: <http://www.ncbi.nlm.nih. gov/pubmed/9872577>. Acesso em: 20 out. 2010. doi: 10.1093/ ps/77.12.1766.

ESTEVEZ, I. et al. Use of cool perches by broiler chickens. Poultry Science, v.81, p.62-69, 2002. Disponível em: <http://www.ncbi.nlm. nih.gov/pubmed/11885901>. Acesso em: 30 mai 2011.

EUROPEAN COMISSION. Health e Consumer Protection Directorate-General. The Welfare of chickens kept for meat production (broilers). Report for the Scientific Committee on Animal Health and Animal Welfare, 2000. Disponível em: <http://ec.europa.eu/food/plant/protection/evaluation/existactive/ list_chlorpropham.pdf>. Acesso em: 04 fev. 2011.

GRANDIN, T. Poultry slaughter plant and farm audit: critical control points for bird welfare, 2009. Disponível em: <http:// www.grandin.com/poultry.audit.html>. Acesso em: 22 fev. 2011.

GREENE, J.A. et al. A contact dermatitis of broilers, clinical and pathological findings. Avian Pathology, v.14, p.2338, 1985. Disponível em: <http://www.tandfonline.com/doi/ abs/10.1080/03079458508436205\#.U2LWbfldXGA>. Acesso em: 01 nov. 2011. doi: 10.1080/03079458508436205.

GROSS, W.B.; SIEGEL, H.S. Evaluation of the heterophil:lymphcyte ratio as a measure of stress in chickens. Avian Diseases, v.27, n.4, p.972-979, 1983. Disponível em: <http://www.ncbi.nlm.nih.gov/pubmed/6360120>. Acesso em: 09 jul. 2010. doi: 10.2307/1590198.

HEIKKILÄ, M. et al. Development of perching in chicks reared in enriched environment. Applied Animal Behaviour Science, v.99, p.145-156, 2006. Disponível em: <http://www.sciencedirect. 
com/science/article/pii/S0168159105003278>. Acesso em: $15 \mathrm{dez}$ 2010. doi: 10.1016/j.applanim.2005.09.013.

JONES, T.A. et al. Environmental and management factors affecting the welfare of chickens on commercial farms in the United Kingdom and Denmark stocked at five densities. Poultry Science, v.84, p.1155-1165, 2005. Disponível em: <http://www. ncbi.nlm.nih.gov/pubmed/16156197>. Acesso em: 23 mar 2010. doi: $10.1093 / \mathrm{ps} / 84.8 .1155$.

MACGEOWN, D. et al. Effect of carprofen on lameness in broiler chicken. Veterinary Record, v.144, p.668-671, 1999. Disponível em: <http://www.ncbi.nlm.nih.gov/pubmed/10404606>. Acesso em: 10 nov. 2010. doi: 10.1136/vr.144.24.668.

PICKEL, T. et al. Perch material and diameter affects particular perching behaviours in laying hens. Applied Animal Behaviour Science, v.27, p.37-42, 2010. Disponível em: <http://www. sciencedirect.com/science/article/pii/S0168159110002078> Acesso em: 10 nov 2010. doi: 10.1016/j.applanim.2010.08.005.

SANOTRA, G.S. et al. Monitoring leg problems in broilers: a survey of commercial broiler production in Denmark. World's Poultry Science Journal, v.57, p.55-69, 2001. Disponível em: <http://journals.cambridge.org/action/displayAbstract?fromPage= online\&aid=622108> . Acesso em: 09 fev. 2011. doi: http://dx.doi. org/10.1079/WPS20010006.
SIMSEK, U.G. et al. Effects of enriched housing design on broiler performance, welfare, chicken meat composition and serum cholesterol. Acta Veterinaria BRNO, v.78, p.67-74, 2009. Disponível em: <http://actavet.vfu.cz/78/1/0067/>. Acesso em: 15 ago. 2010. doi: 10.2754/avb200978010067.

SHEPHERD, E.M.; FAIRCHILD, B.D. Footpad dermatitis in poultry. Poultry Science, v.89, p.2043-2041, 2010. Disponível em: <http://www.ncbi.nlm.nih.gov/pubmed/20852093>. Acesso em: 07 fev. 2011. doi: 10.3382/ps.2010-00770.

STRUELENS, E. et al. Perch width preferences of laying hens. British Poultry Science, v.50, n.4, p.418423, 2009. Disponível em: <http://www.ncbi.nlm.nih. gov/pubmed/19735010>. Acesso em: 04 set. 2010. doi: $10.1080 / 00071660903110885$.

SU, G. et al. A note on the effects of perches and litter substrate on leg weakness in broiler. Poultry Science, v.79, p.125912663, 2000. Disponível em: <http://www.ncbi.nlm.nih.gov/ pubmed/11020069>. Acesso em: 22 fev. 2011

VESTERGAARD, S.; SANOTRA, G.S. Relationships between leg disorders and changes in the behavior of broiler chickens. Veterinary Record, v.144, p.205-209, 1999. Disponível em: <http://www.ncbi.nlm.nih.gov/pubmed/10097343>. Acesso em: 02 fev. 2011. doi: 10.1136/vr.144.8.205. 